\title{
$\cup \quad$ Australian age-sex-specific COVID-19 mortality in international comparative perspective, to June 2020
}

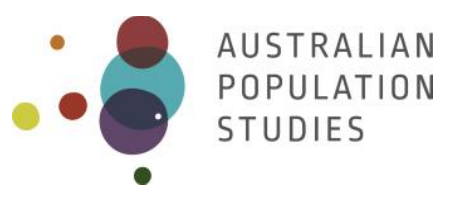

\author{
Rebecca Kippen* Monash University \\ * Email: rebecca.kippen@monash.edu. Address: School of Rural Health, Monash University, \\ 26 Mercy Street, Bendigo, Victoria, Australia.
}

Paper received 28 July 2020; accepted 9 November 2020; published 16 November 2020

\section{Introduction}

By the end of June 2020, a combination of good luck and good management had resulted in Australia having one of the lowest COVID-19 death rates in the developed world at that time. Australia's success was heralded nationally and internationally (Duckett and Stobart 2020; Bremmer 2020). The second wave of COVID-19 deaths that occurred in Australia from July to October 2020 was, in June 2020 , only a possible threat.

At 30 June 2020, Australia had recorded 103 deaths from COVID-19, with only 1 death reported in June. This translates to 4 deaths per million population. Developed countries with similarly low COVID-19 death rates (under 10 deaths per million population), January-June 2020, included Japan (8), Brunei (7), South Korea (6), Slovakia (5), Singapore (4), and New Zealand (4) (calculated from WHO 2020; United Nations 2019).

In contrast, the following 11 developed countries had recorded COVID-19 death rates more than 50 times higher than that of Australia (above 200 deaths per million population), as at 30 June 2020: Belgium (842), United Kingdom (644), Spain (606), Italy (575), Sweden (528), France (456), United States (382), Netherlands (357), Ireland (352), Chile (298), and Canada (227) (calculated from WHO 2020; United Nations 2019). Deaths and death rates were still rising strongly in several of these countries in mid-2020.

Despite vastly differing levels of mortality, age-sex patterns of COVID-19 deaths appear to be consistent internationally (at least for those countries with available data). Death rates are higher for males than for females, low in childhood and young adulthood, and increase markedly through middle and old age.

\section{Data and methods}

This study is based on age-sex-specific COVID-19 death rates for the first half of 2020 for 7 developed countries with particularly low or high COVID-19 mortality and for which consistent age-sex death data could be sourced. These 7 countries are Australia, Japan, South Korea, Belgium, England \& Wales (constituting $89 \%$ of the United Kingdom's population), Italy, and the Netherlands. 
Counts of deaths by age and sex were sourced from national governments for Australia (AGDH 2020) and England and Wales (ONS 2020), and from COVerAGE-DB (Riffe et al. 2020) for the remaining 5 countries. Population estimates by age and sex for 2020 were sourced from World Population Prospects (United Nations 2019). Death counts and population estimates were used to calculate COVID-19 death rates for each population by sex for age groups 0-49 (a broad grouping since COVID19 mortality under the age of 50 years was relatively low for all considered populations), 50-59, 60-69, 70-79, 80-89, and 90+ years.

To show the age-sex COVID-19 mortality pattern for each population, taking into account the enormously disparate death-rate levels, rate ratios were used. For each country, the ratio was calculated of the mortality rate for each age-sex category to the mortality rate for males aged $90+$ years (the highest age-sex rate for every country considered). These ratios are shown in Figure 1.

Age-standardised mortality rates for each sex for each country were calculated using Australia's 2020 population by age as the standard. These were used to calculate the male-to-female ratio of agestandardised COVID-19 mortality for each country, as a summary measure of excess male mortality, shown in Figure 2.

\section{Key features}

Figure 1 shows the age-sex pattern of COVID-19 mortality for each country. Figure 1a shows 4 populations that have remarkably similar patterns, despite the fact that the overall mortality of 2 of the populations (Belgium, and England \& Wales) was 100-200 times higher than that of the other 2 countries (Australia and South Korea). For all 4 populations, female COVID-19 mortality at age 90+ years was $64-72 \%$ that of males of the same age. Male and female mortality at age 80-89 years was respectively $34-41 \%$ and $23-27 \%$ of male mortality at age $90+$ years. Younger age groups show similar clustered differentials at lower levels.

Figure $1 \mathrm{~b}$ displays the remaining 3 populations - Japan, Italy, and the Netherlands (with Australia as a comparator) - with dissimilar age-sex patterns of COVID-19 mortality, although they all exhibit higher male mortality and a positive correlation of mortality with age. Japanese and Italian male mortality for age groups under 90 years is relatively high compared to the patterns seen in Figure 1a, while Japanese female mortality at age $90+$ years is remarkably low relative to Japanese male mortality at the same age. Female mortality at older ages for Italy and the Netherlands is relatively high.

Ratios of male-to-female age-standardised COVID-19 death rates for each country are shown in Figure 2 in order of magnitude. In Belgium, Australia, Netherlands, England \& Wales, and South Korea, age-standardised COVID-19 mortality for males was $61-73 \%$ higher than for females. In contrast, COVID-19 mortality for Italian males was more than twice that for females, while Japanese male mortality was almost 3 times female mortality. These 7 country-level sex ratios are much higher than those seen for the most similar cause-of-death groupings. For example, for Australia in 2019, age-standardised male mortality for Certain infectious and parasitic diseases was only $16 \%$ higher than female mortality, and for Influenza and pneumonia 11\% higher (ABS 2020). 

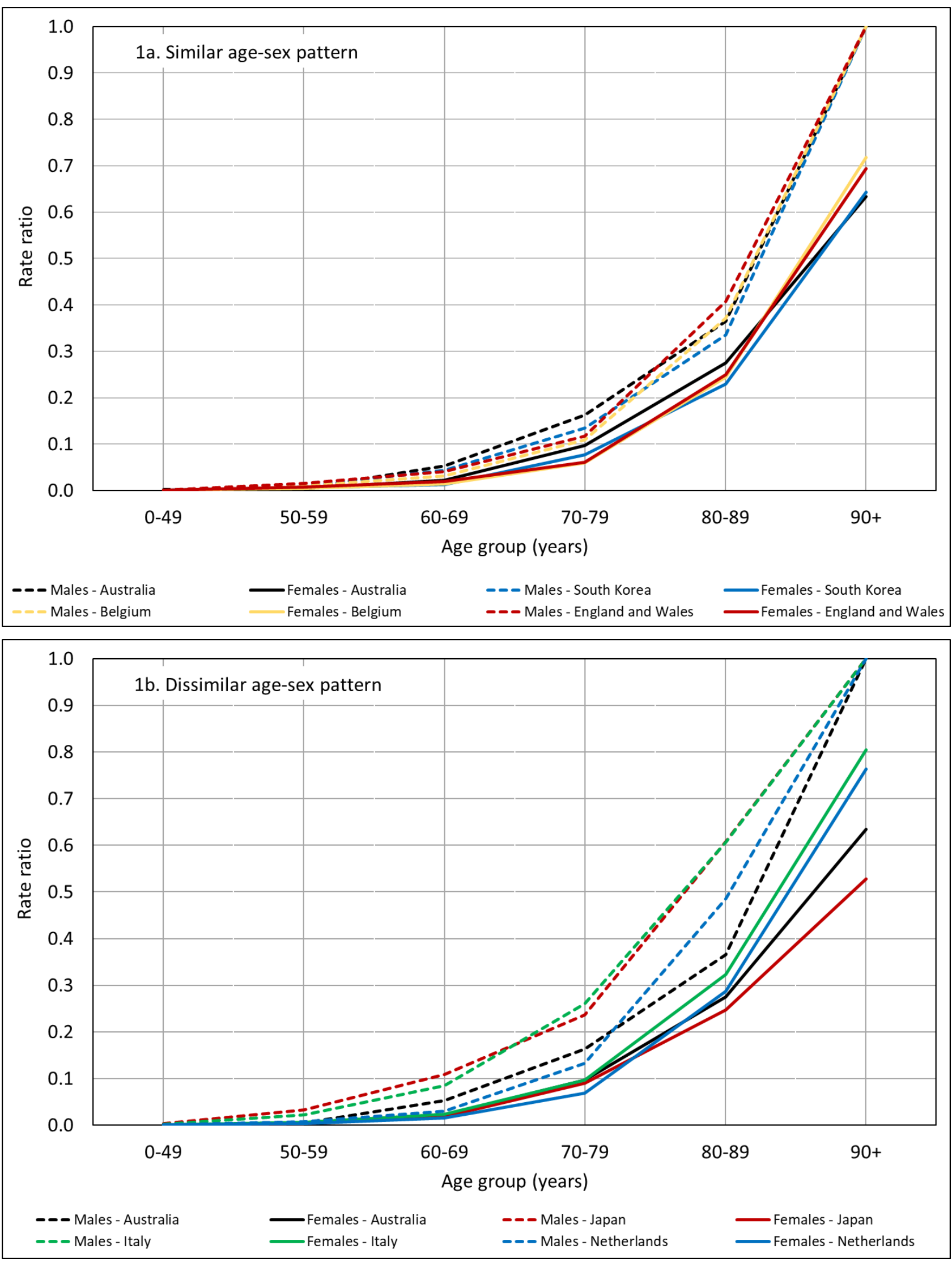

Figure 1: Similar (1a) and dissimilar (1b) age-sex patterns of COVID-19 mortality (rate ratio to mortality for males age 90+ years), selected developed countries, January-June 2020 


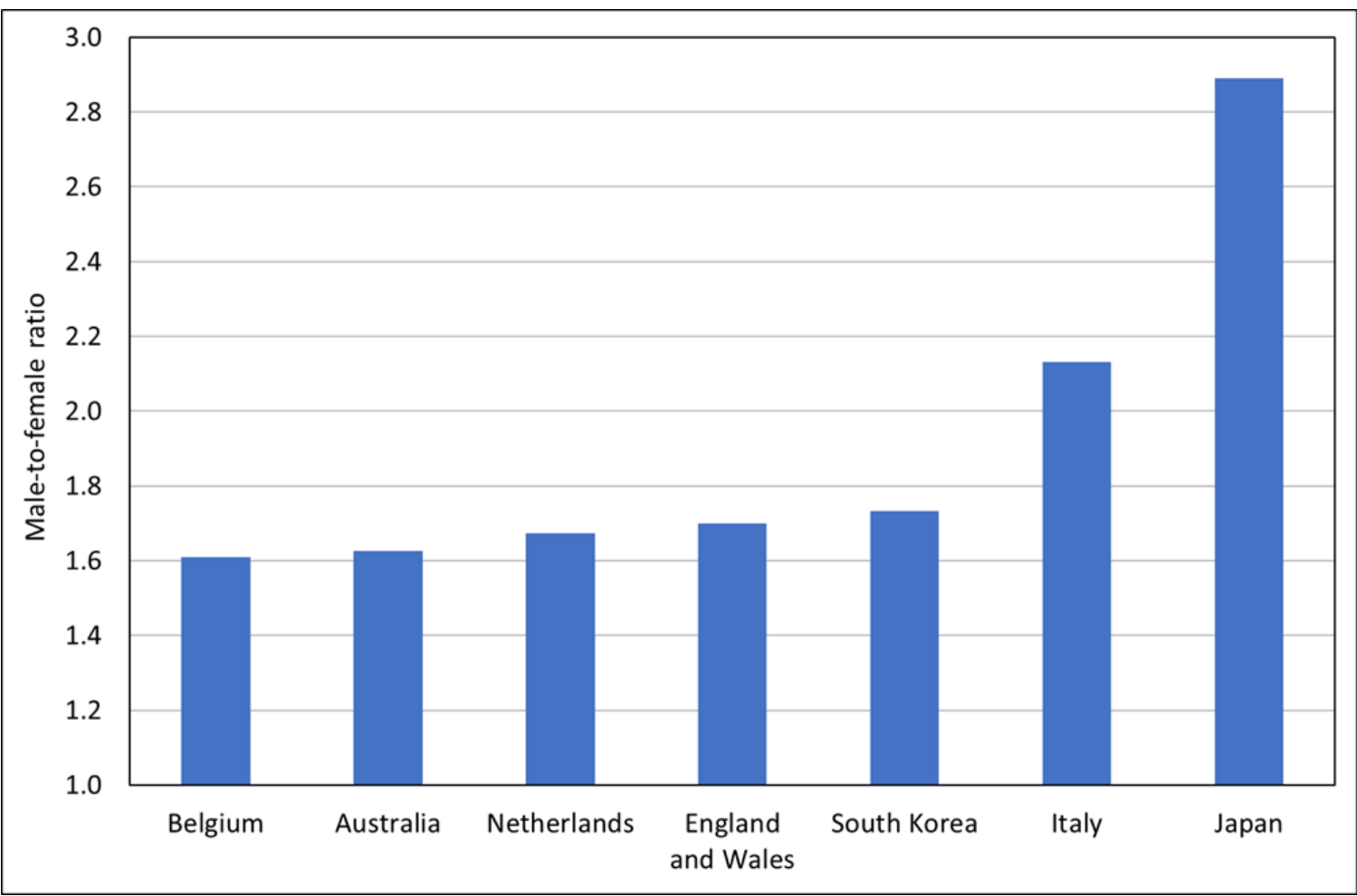

Figure 2: Male-to-female ratio of age-standardised COVID-19 mortality, selected developed countries, January-June 2020

What explains this disparity in sex-specific COVID-19 mortality? Early research indicates that females produce a more effective immune response to SARS-CoV-2 (the coronavirus that causes COVID-19) than do males, and that this response declines across age for both sexes (Takahashi et al. 2020). In addition, co-morbidities (such as hypertension) and risk behaviours (such as smoking and drinking) that are more prevalent in males may play a role in higher male COVID-19 mortality (Gebhard et al. 2020).

Areas for future research include the factors behind non-standard age-sex patterns of COVID-19 death rates, such as those seen for Japan and Italy, and reasons for the much higher male-to-female mortality ratios for these countries. In addition, future research will reveal whether the second wave of COVID-19 deaths in Australia and other countries results in similar age-sex mortality patterns to those seen in the first half of 2020 , or whether and why there are substantive differences.

\section{Limitations}

Although death registration in each country considered is complete, or near complete, COVID-19 death rates may be inaccurate because of (a) mis-estimates of the 2020 denominator population, or, more importantly (b) mis-recording of COVID-19 as a cause of death. 'Excess deaths' in 2020 in many countries greatly exceed reported COVID-19 deaths (Felix-Cardoso et al. 2020; The Economist 2020). This indicates that COVID-19 as a cause of death is under-recorded, and that therefore the death rates discussed above, are, if anything, underestimates. This could also be the case in Australia (Bennett 2020). The extent of underestimation may vary by age and sex, affecting the age-sex patterns described above. 


\section{Ethics approval}

This study was approved by the Monash University Human Research Ethics Committee, project ID 25067.

\section{Acknowledgements}

The author thanks the editor and two anonymous reviewers whose helpful suggestions improved this work. Most data are derived from COVerAGE-DB: A Database of COVID-19 Deaths by Age (led by researchers at the Max Planck Institute for Demographic Research), World Population Prospects (United Nations) and Coronavirus Disease (COVID-19) Situation Reports (World Health Organization). The author thanks the respective research teams for assembling these international data and making them publicly available under creative commons licenses.

\section{References}

Australian Bureau of Statistics (ABS) (2020) Causes of Death, Australia, 2019, catalogue number 3303.0. https://www.abs.gov.au/statistics/health/causes-death/causes-deathaustralia/2019/3303 1\%20Underlying\%20causes\%20of\%20death\%20\%28Australia\%29.xlsx. Accessed 8 November 2020.

Australian Government Department of Health [AGDH] (2020) COVID-19 Deaths by Age Group and Sex, 1 July 2020. https://www.health.gov.au/resources/covid-19-deaths-by-age-group-and-sex. Accessed 2 July 2020.

Bennett C (2020) Have there been uncounted coronavirus deaths in Australia? We can't say for sure, but the latest ABS data holds clues. The Conversation, 26 June 2020. https://theconversation.com/have-there-been-uncounted-coronavirus-deaths-in-australia-wecant-say-for-sure-but-the-latest-abs-data-holds-clues-141363. Accessed 2 July 2020.

Bremmer I (2020) The best global responses to COVID-19 pandemic. Time, 12 June 2020. https://time.com/5851633/best-global-responses-covid-19/. Accessed 8 November 2020.

Duckett $S$ and Stobart A (2020) 4 ways Australia's coronavirus response was a triumph, and 4 ways it fell short. The Conversation, 4 June 2020. https://theconversation.com/4-ways-australiascoronavirus-response-was-a-triumph-and-4-ways-it-fell-short-139845. Accessed 8 November 2020.

Felix-Cardoso J, Vasconcelos H, Rodrigues P, and Cruz-Correia R (2020) Excess mortality during COVID-19 in five European countries and a critique of mortality analysis data. medRxiv. https://www.medrxiv.org/content/10.1101/2020.04.28.20083147v1. Accessed 3 July 2020.

Gebhard C, Regitz-Zagrosek V, Neuhauser H K, Morgan R, and Klein S L (2020) Impact of sex and gender on COVID-19 outcomes in Europe. Biology of Sex Differences 11(2): 1-13.

Office for National Statistics (ONS) (2020) Number of deaths registered each month in England and Wales, including deaths involving the coronavirus (COVID-19), by age, sex and country. https://www.ons.gov.uk/peoplepopulationandcommunity/birthsdeathsandmarriages/deaths/dat asets/deathsinvolvingcovid19englandandwales. Accessed 1 November 2020.

Riffe T, Acosta T, et al. (2020) COVerAGE-DB: A Database of COVID-19 Deaths by Age. https://osf.io/mpwjq/. Accessed 7 November 2020.

Takahashi T, Ellingson M K, Wong P, Israelow B et al. (2020) Sex differences in immune responses that underlie COVID-19 disease outcomes. Nature. https://doi.org/10.1038/s41586-020-2700-3. Accessed 8 November 2020. 
The Economist (2020) Tracking covid-19 excess deaths across countries.

https://www.economist.com/graphic-detail/2020/04/16/tracking-covid-19-excess-deaths-acrosscountries. Accessed 2 July 2020.

United Nations (2019) Country-level populations, totals, and by age and sex. World Population Prospects. https://population.un.org/wpp/. Accessed 2 July 2020.

World Health Organization (WHO) (2020) Coronavirus Disease (COVID-19) Situation Report 163, 1 July 2020. https://www.who.int/emergencies/diseases/novel-coronavirus-2019/situation-reports. Accessed 2 July 2020 